\title{
Quantitative Comparison of Manual vs. 3D Scanner Human Body Measurements
}

\author{
Andrew KOVAL* \\ Bremen University, Bremen, Germany \\ https://doi.org/10.15221/20.35
}

\begin{abstract}
Human body measurements are actively used in the clothing industry for production or finding the best possible garment fit. For the last few years digital methods based on 3D scans are replacing the traditional manual measurement techniques. In this paper we determine the quality of measurements obtained with a 3D scanner in terms of reliability and validity. Tape measurements from two expert measurers were used as a reference. Reliability comparison was evaluated in terms of Standard Error of Measurement and difference from the mean distribution. Validity was analyzed according to ISO 20685.

Data used for the analysis were gathered during Phase 2 of "Comparative Analysis of measurement methods of 3D body scanning" organized by IEEE Industry Connections 3D Body Processing group hosted in Instituto de Biomecánica de Valencia (IBV).

For all 3D scanner measurements $90 \%$ of differences from the mean are inside the ANSUR (Anthropometric Survey of US Army Personnel) allowable error interval. It was also shown that reliability of measurements obtained with Texel Portal MX 3D scanner is nearly twice better than reliability of manual measurements. Upon application of constant offsets these two measurement techniques are compatible according to ISO 20685.
\end{abstract}

Keywords: 3D body scanning, human body measurements, comparative analysis

\section{Introduction}

Body measurements estimation is an important task in garment production and best fit garment determination. Traditionally in the clothing industry measurements were captured with tape measures, but nowadays digital methods based on 3D scans are replacing the traditional measurement techniques. Advantage of the scanner-based methods is that they allow getting hundreds of measurements in just a few minutes in an automatic way using the scanned surface of the human body.

However, traditional manual measurements often use palpation of the bones underneath the skin in order to identify anthropometric landmarks. For example, according to ISO 8559 [1] waist girth should be measured at the level of midway between the lowest rib point and the highest point of the hip bone. As 3D scan of a body is just a digital imprint of the surface it is quite a challenge to determine these anthropometric points on it [2]. However several scientific groups show competitive to traditional measurements results $[3,4]$.

In this research we compared traditional measurements and measurements derived by Texel Portal MX scanner in terms of reliability and validity.

Data for analysis was gathered during Phase 2 of "Comparative Analysis of measurement methods of 3D body scanning", $14^{\text {th }}$ to $18^{\text {th }}$ October 2019, Valencia (Spain), organized by IEEE Industry Connections 3D Body Processing group [5] hosted in Instituto de Biomecánica de Valencia (IBV). Eight measurements stations participated in this survey including Texel Portal MX scanner and two measurement experts. Traditional measurement experts have completed special training before the survey and are experienced with ISO 8559 techniques.

\section{Materials and Methods}

\subsection{Equipment}

Texel Portal MX scanner consists of a rotating platform and a stand with four depth cameras.

The Texel Portal MX is a full color 3D body scanner. It consists of a rotating platform, a column with 4 depth sensors (Asus Xtion Pro Live) and high-performance computer. A person can be fully 3D scanned in 30 seconds and a detailed 3D preview model can be viewed in 1 minute. Before scanning each participant was instructed to stand in the center of the platform, take an A-pose, look straight and not to move. During scanning the platform made two full turns. As a result a 3D model was constructed and its preview was shown on the screen.

*andriiko@gmail.com 


\subsection{Participants}

Subject sample consisted of 72 adult healthy subjects: 36 females and 36 males aged from 18 to 57 years, BMI ranged from 17.7 to $42.8 \mathrm{~kg} / \mathrm{m}^{2}$. Each participant was measured twice by each station, so there were four repetitions of manual measurements (2 experts * 2 repetitions) and two repetitions of Texel Portal MX scanner measurements.
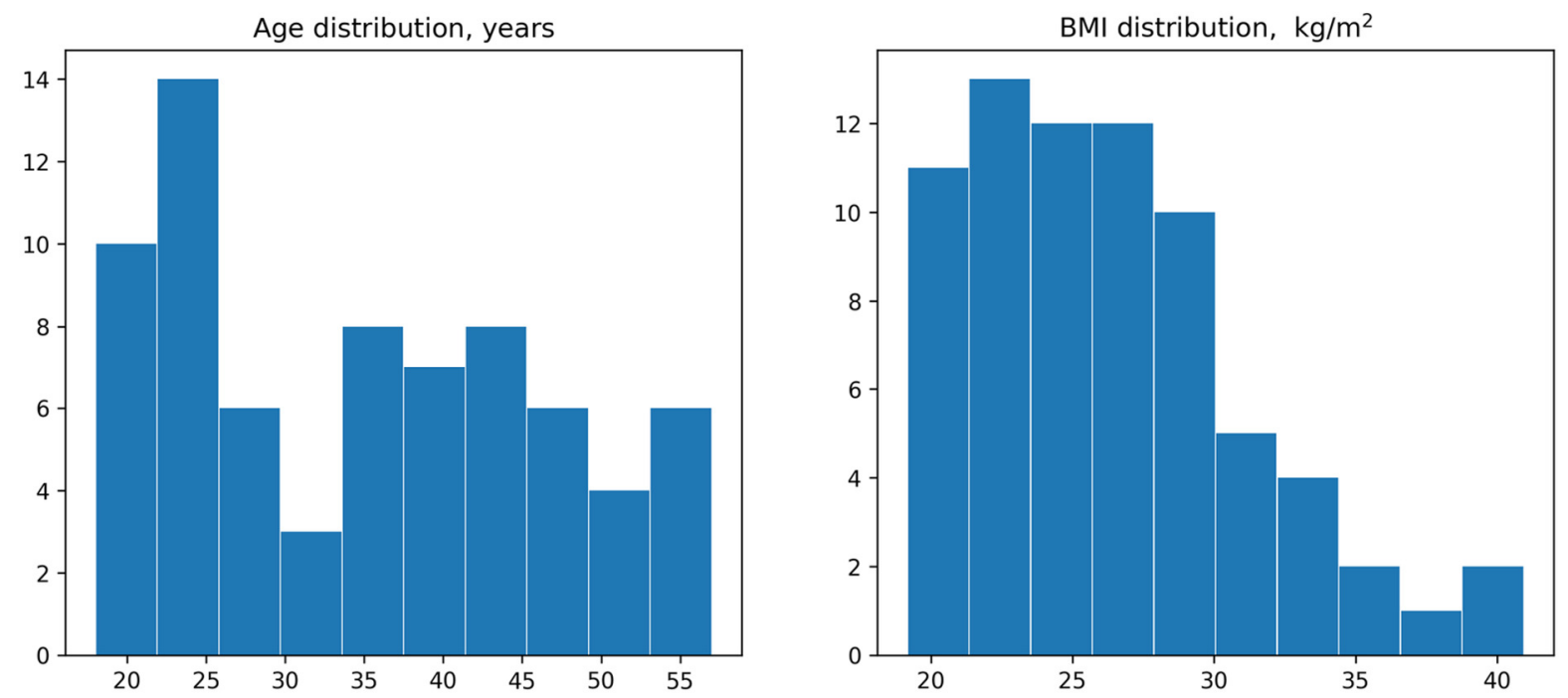

Fig. 1. Participants age and BMI distribution.

\subsection{Body measurements}

14 measurements were obtained from Texel Portal MX scanner and 12 measurements from traditional measurers. Manual measurements were taken from the right side only for symmetrical measurements while scanner measurements were taken on both sides. Lowaist [6] was not implemented in Texel Portal MX scanner and it was taken only by manual measurers. Only measurements present both in scanner and manual datasets were used in comparison.

\begin{tabular}{|l|c|c|c|c|}
\hline Measurement name & Body side & Units & ISO 8559-1 (2017) & Measurement station \\
\hline Neck girth & - & $\mathrm{mm}$ & 5.3 .2 & Scanner and manual \\
\hline Back neck point to waist & - & $\mathrm{mm}$ & 5.4 .5 & Scanner and manual \\
\hline Upper arm girth R & right & $\mathrm{mm}$ & 5.3 .16 & Scanner and manual \\
\hline Upper arm girth L & left & $\mathrm{mm}$ & 5.3 .16 & Only scanner \\
\hline Back neck point to wrist R & right & $\mathrm{mm}$ & 5.4 .17 & Scanner and manual \\
\hline Back neck point to wrist L & left & $\mathrm{mm}$ & 5.4 .17 & Only scanner \\
\hline Across back shoulder width & - & $\mathrm{mm}$ & 5.4 .3 & Scanner and manual \\
\hline Bust girth & - & $\mathrm{mm}$ & 5.3 .4 & Scanner and manual \\
\hline Waist girth & - & $\mathrm{mm}$ & 5.3 .10 & Scanner and manual \\
\hline Hip girth & - & $\mathrm{mm}$ & 5.3 .13 & Scanner and manual \\
\hline Thigh girth R & right & $\mathrm{mm}$ & 5.3 .20 & Scanner and manual \\
\hline Thigh girth L & left & $\mathrm{mm}$ & 5.3 .20 & Only scanner \\
\hline Total crotch length & - & $\mathrm{mm}$ & 5.4 .18 & Scanner and manual \\
\hline Inside leg height & - & $\mathrm{mm}$ & 5.1 .15 & Scanner and manual \\
\hline Lowaist [6] & - & $\mathrm{mm}$ & - & Only manual \\
\hline
\end{tabular}

Table 1. Measurements obtained. 


\section{Data processing}

Measurements were loaded and cleaned from outliers according to ISO 15535 [7]. As detailed below, only outliers of manual measurements were cleaned, obtained scanner measurements have no outliers. Mean values and standard deviations were calculated for each measurement and measurement station. All measurements that fell outside of 3 standard deviations were investigated. For each measurement station and each measurement correlation scatter plots were built, where axes correspond to measurement values of the first and the second measurement repetitions. There were 33 correlation scatter plots built and analyzed in total ( 3 stations * 11 measurements). If any correlation plot showed uncorrelated measurements for the subject, then both uncorrelated measurements were compared to other stations results. This procedure allowed not to discard both uncorrelated measurements but to detect only one outlier and discard it.

As a result eight abnormal values were detected and discarded as per this outlier removal standard. Since dataset size used for the analysis is equal to 4752 (72 participants * 11 measurements * 3 stations * 2 repetitions), number of outliers is less than a percent of the dataset size. It can be noticed that outliers were only in manual measurements.

\begin{tabular}{|c|c|c|c|c|}
\hline № & Subject & Measurement name & Station & Repetition \\
\hline 1 & IEEEP2_30 & Neck girth & Manual measurer 2 & 2 \\
\hline 2 & IEEEP2_18 & Upper arm girth R & Manual measurer 1 & 1 \\
\hline 3 & IEEEP2_70 & Upper arm girth R & Manual measurer 1 & 2 \\
\hline 4 & IEEEP2_55 & Bust girth & Manual measurer 1 & 1 \\
\hline 5 & IEEEP2_70 & Waist girth & Manual measurer 2 & 1 \\
\hline 6 & IEEEP2_28 & Waist girth & Manual measurer 2 & 2 \\
\hline 7 & IEEEP2_55 & Total crotch length & Manual measurer 1 & 1 \\
\hline 8 & IEEEP2_53 & Inside leg height & Manual measurer 1 & 1 \\
\hline
\end{tabular}

Table 2. Outliers discarded.

\section{Reliability}

Reliability analysis was performed in terms of Standard Error of Measurement (SEM) [8] and comparison of absolute deviations of measurements to ANSUR [9] allowable errors.

SEM was calculated for each station separately. The results are shown on Fig. 2. For all measurements scanner SEM doesn't exceed individual expert errors with the only exception of "Across back shoulder width" measurement where scanner error is bigger than manual errors. It was caused by variation in shoulders position in A-pose due to inconsistent instructions regarding posture during survey. To set correct shoulders position participants should be instructed to relax their arms and shoulders and then to raise arms to the required height without moving shoulders. This can help to increase reliability of such measurements as "Across back shoulder width" and "Back neck point to wrist R", which depend on shoulders position. According to Fig. 2 it can be said that scanner measurements are in average more reliable than any individual manual measurer.

Differences from the mean for all manual measurements are shown on Fig. 3. Each point on the plot represents a difference of a particular manual measurement from a mean of four manual measurement repetitions (each of two measurers have measured twice) for a particular participant. So here we compare not station reliability but technique reliability.

Almost for all manual measurements $95 \%$ data points interval is out of bounds of ANSUR allowable error. Moreover, for "Back neck point to waist", "Back neck point to wrist R" and "Upper arm girth R" manual measurements more than $50 \%$ data points are outside of the allowable error interval. The only manual measurement for which $95 \%$ of the points are inside of the ANSUR allowable error interval is "Across back shoulder width".

Such big measurement dispersion relatively to ANSUR allowable errors may be explained by the fact that there were no strict requirements for manual measurements precision in the survey used for this analysis. This survey objective was to compare different measurement techniques in their routine regime. 
Fig. 4 represents the same plot but for the scanner results. Data is shown on the same scale as in the previous figure. For all scanner measurements $90 \%$ of differences from the mean are inside the ANSUR allowable error interval. Moreover, for 10 out of 11 measurements $95 \%$ of differences are inside the allowable interval. And for measurements "Bust girth", "Waist girth", "Hip girth", "Total crotch length" and "Inside leg height" 95\% interval is nearly twice as small as allowable error.

Comparing Fig. 3 and Fig. 4 it can be concluded that scanner measurements reliability is nearly twice better than reliability of measurements taken by multiple manual measurers.

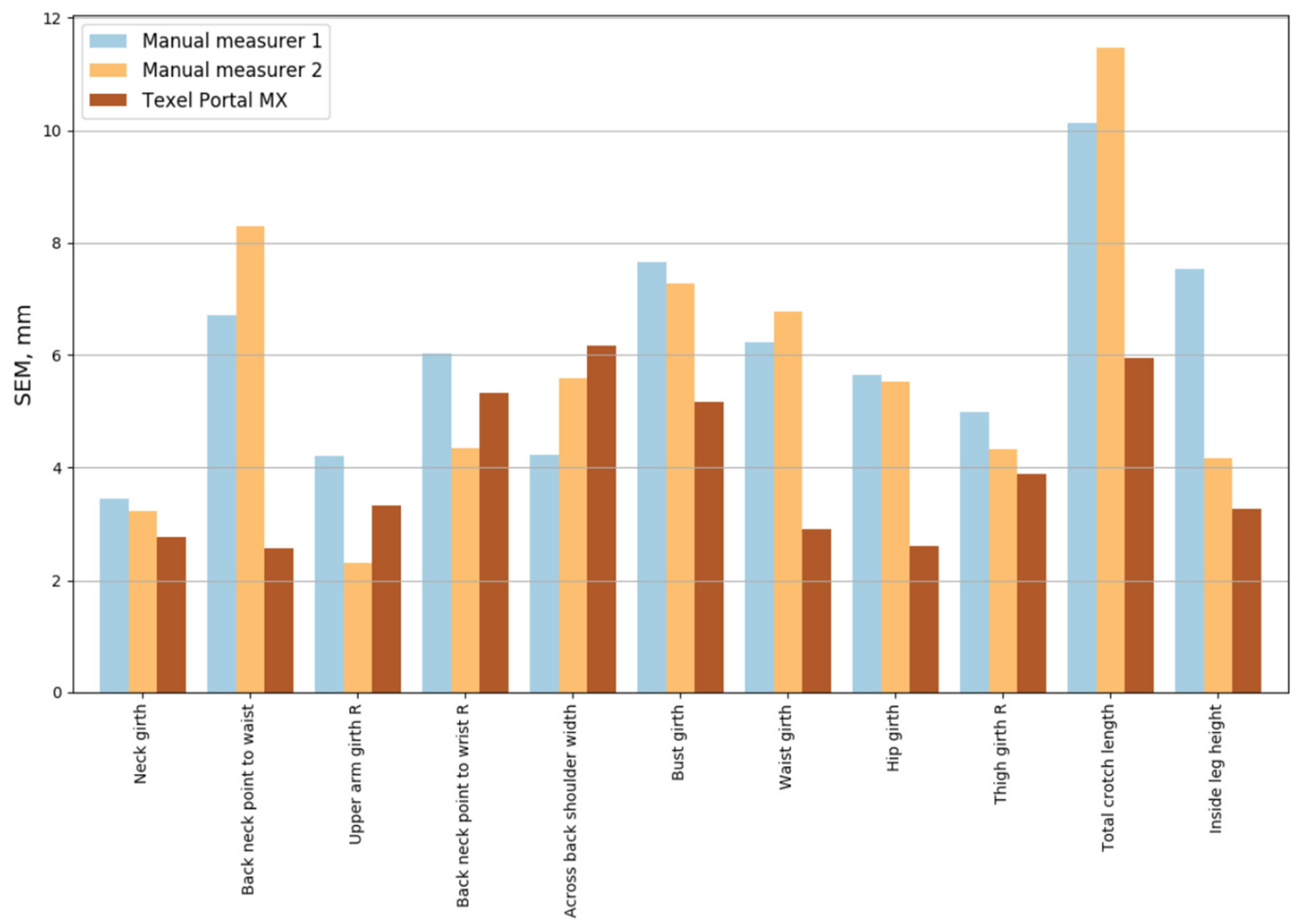

Fig. 2. Standard Error of Measurement comparison. 


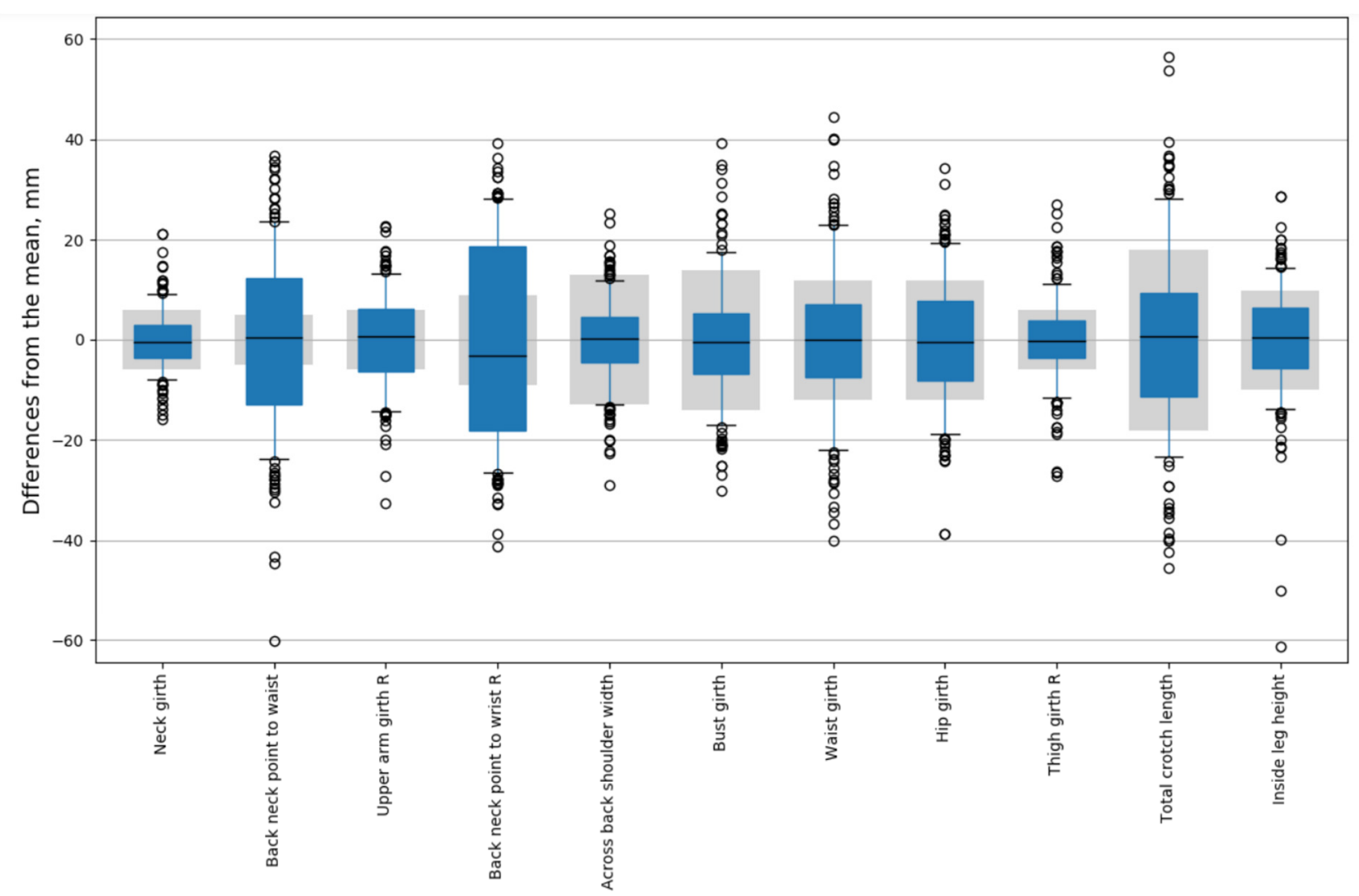

Fig. 3. Differences from the mean distribution for manual measurements. Grey boxes are ANSUR allowable errors, blue boxes contain $50 \%$ of data points, whiskers contain $95 \%$ of data points. Small circles represent most distant $5 \%$ measurements from the median.

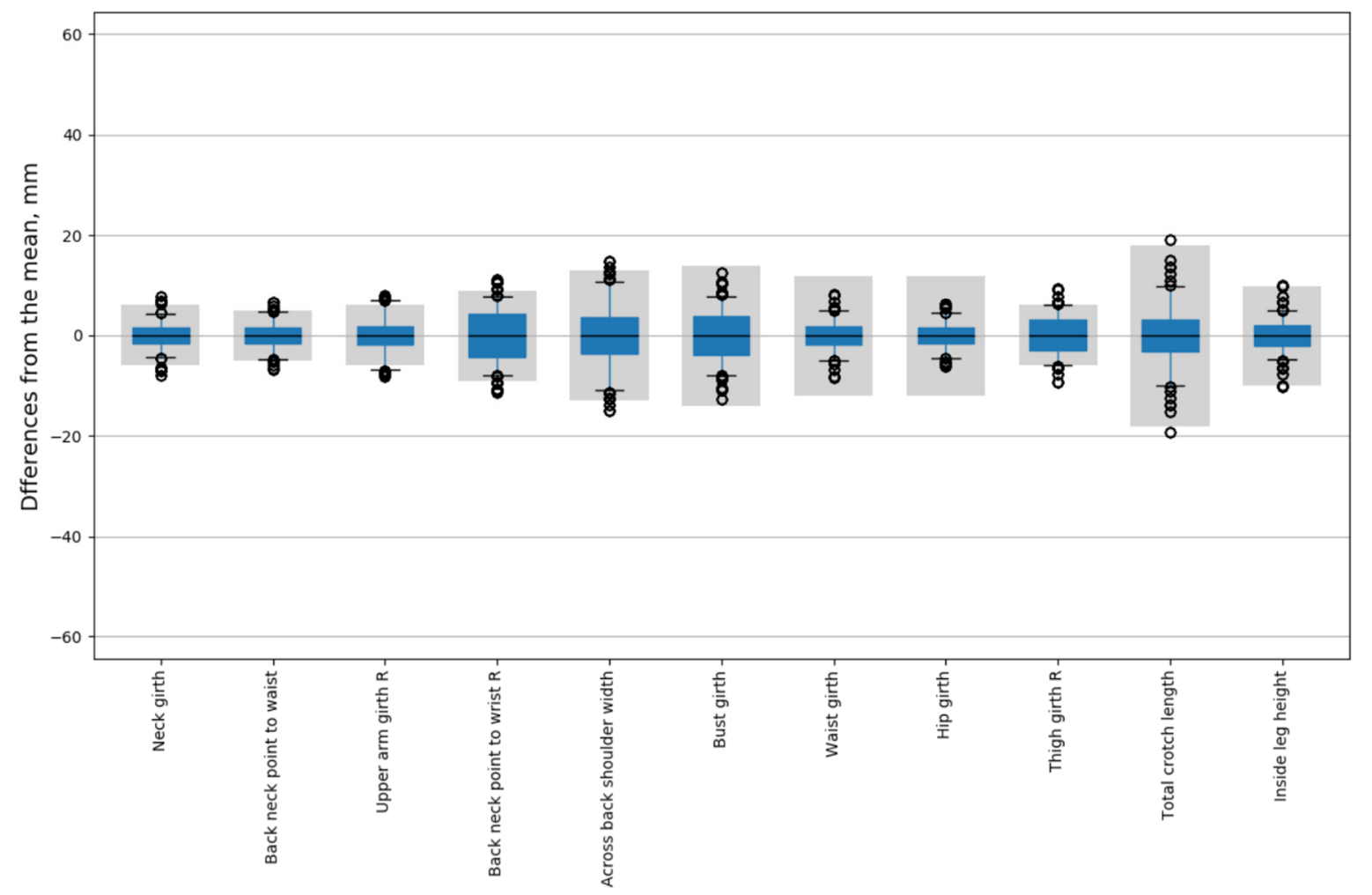

Fig. 4. Differences from the mean distribution for scanner measurements. Grey boxes are ANSUR allowable errors, blue boxes contain $50 \%$ of data points, whiskers contain $95 \%$ of data points. Small circles represent most distant 5\% measurements from the median. 


\section{Validity}

Validity analysis was performed according to ISO 20685 [10] validity algorithm.

All combinations of scanner minus manual measurements were computed for each person. There were two scanner measurements and four manual measurements in the dataset, which corresponds to eight differences for each measurement and each person. The mean of these differences with its associated standard deviation ( $\sigma$ ), sample size, 95\% confidence interval (Cl) and ISO 20685 allowable error are reported in Table 3.

\begin{tabular}{|l|c|c|c|c|c|}
\hline \multicolumn{1}{|c|}{ Measurement } & Mean, $\mathrm{mm}$ & $\sigma, \mathrm{mm}$ & Sample size & $95 \% \mathrm{Cl}, \mathrm{mm}$ & $\begin{array}{c}\text { ISO allowable } \\
\text { error, } \mathrm{mm}\end{array}$ \\
\hline Neck girth & 3.2 & 0.5 & 574 & $(2.3,4.2)$ & 4 \\
\hline Back neck point to waist & -21.5 & 1.0 & 576 & $(-23.4,-19.6)$ & 5 \\
\hline Upper arm girth R & 9.1 & 0.5 & 572 & $(8.2,10.1)$ & 3 \\
\hline Back neck point to wrist R & -19.1 & 1.0 & 576 & $(-21.0,-17.2)$ & 5 \\
\hline Across back shoulder width & 7.4 & 1.0 & 576 & $(5.5,9.3)$ & 5 \\
\hline Bust girth & -0.5 & 0.8 & 574 & $(-2.1,1.1)$ & 9 \\
\hline Waist girth & -0.1 & 0.8 & 572 & $(-1.7,1.4)$ & 9 \\
\hline Hip girth & 16.2 & 0.7 & 576 & $(14.9,17.6)$ & 9 \\
\hline Thigh girth R & -4.9 & 0.6 & 576 & $(-6.0,-3.7)$ & 4 \\
\hline Total crotch length & 18.1 & 1.2 & 574 & $(15.7,20.5)$ & 5 \\
\hline Inside leg height & 11.3 & 0.7 & 574 & $(9.9,12.8)$ & 4 \\
\hline
\end{tabular}

Table 3. Scanner minus manual measurements distribution description.

On the Fig. 5 (a) 95\% confidence intervals of the mean and ISO allowable errors corresponding to the table are shown. According to Table 3 and Fig. 5 (a) 95\% Cl of "Bust girth" and "Waist girth" fall completely inside ISO allowable error, thus "Bust girth" and "Waist girth" scanner measurements and traditional measurements measure the same girths and can be used interchangeably. Other measurements have quite small dispersion to fit the allowable error, but due to the shifted means of the distribution confidence intervals go beyond the allowable error.

When the variability of differences is low but the overall error is big it means that we have a systematic error that leads to a bias in measurements results between two measurement techniques. To compensate for this systematic error common practice is to subtract this constant offset from the output data. This procedure can be considered as calibration. If we do this procedure with our data we will see that all 95\% confidence intervals now fall inside the ISO allowable errors (Fig. 5 (b)) and measurement methods can be considered equivalent from an ISO 20685 perspective. 


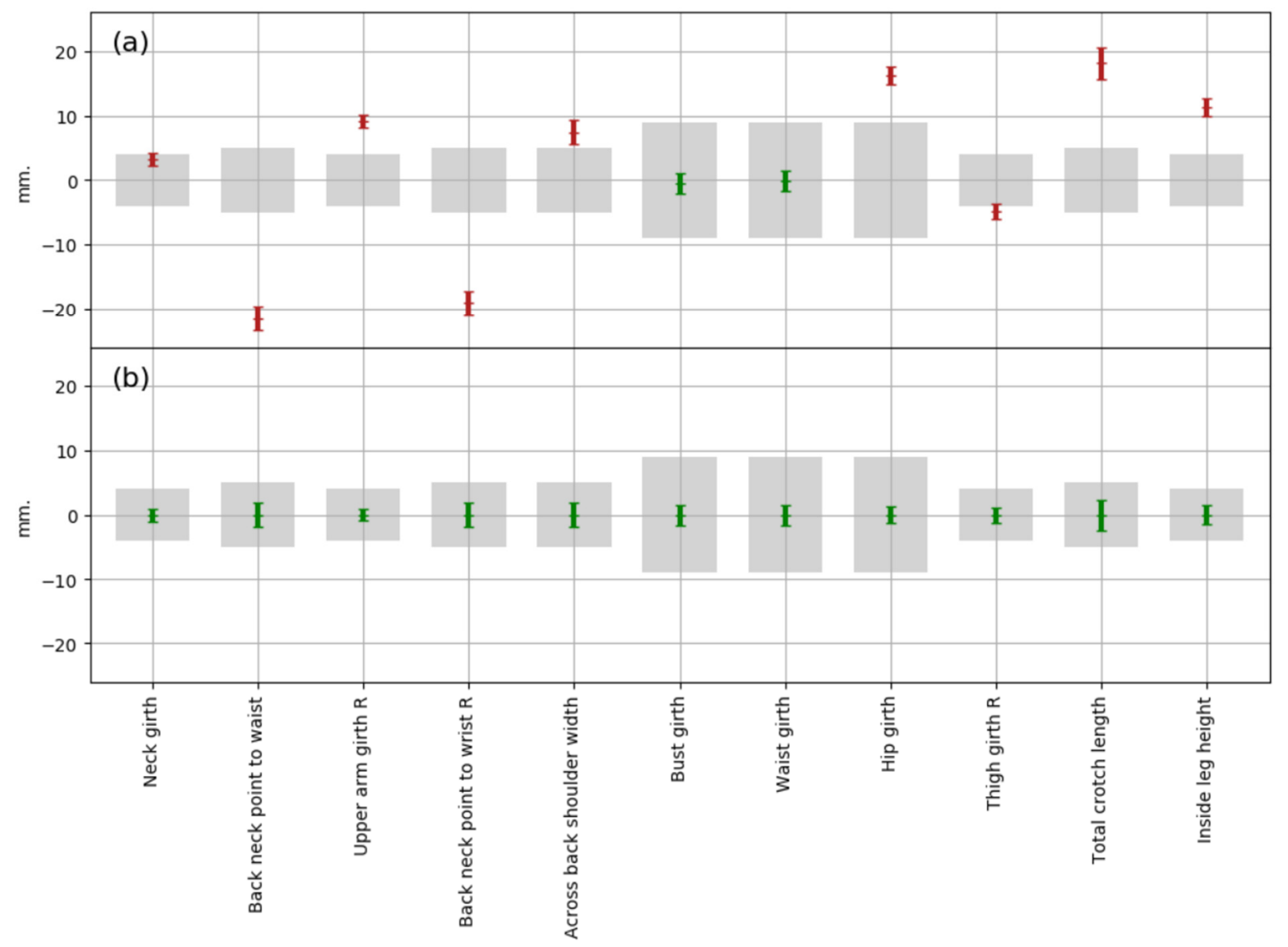

Fig. 5. Scanner minus manual measurement distribution.

(a) - plot without introducing constant offsets, (b) - plot with introduced constant offset. Red and green intervals represent 95th confidence intervals for the mean. Grey boxes - ISO allowable errors. Intervals that are entirely inside ISO allowable error are marked in green, intervals that are not within the allowed error are marked in red.

\section{Conclusions}

As a result of this study, we analyzed reliability and compatibility of scanner and manual measurement techniques.

During data preprocessing eight manual measurements and no scanner measurements were found to be outliers.

We compared SEM for manual measurers and scanner and showed that for almost all measurements scanner reliability is the same or higher than manual measurers reliability.

Additionally reliability of traditional and scanner techniques were compared in terms of ANSUR allowable errors. For all scanner measurements $90 \%$ of differences from the mean are inside the ANSUR allowable error interval.

Furthermore, dispersion of scanner measurements is nearly twice lower than traditional measurements.

Validity analysis was performed based on ISO 20685. According to it scanner and traditional measurement techniques may be considered compatible after accounting for constant offsets.

\section{Acknowledgements}

Authors would like to thank Carol McDonald and IEEE 3D Body Processing Group for organizing the survey and Alfredo Ballester from IBV for managing the survey and for the dataset provided. 


\section{References}

[1] ISO 8559-1:2017 Size designation of clothes - Part 1: Anthropometric definitions for body measurement.

[2] S. Gill, C. J. Parker, S. Hayes, K. Brownbridge, P. Wren, and A. Panchenko, "The True Height of the Waist: Explorations of Automated Body Scanner Waist Definitions of the TC2 scanner", In 5th International Conference on 3D Body Scanning Technologies, 2014, pp. 55-65.

[3] Ballester et al., "3D Human Models from 1D, 2D \& 3D Inputs: Reliability and Compatibility of Body Measurements", in Proc. of 3DBODY.TECH 2018 - 9th Int. Conf. and Exh. on 3D Body Scanning and Processing Technologies, Lugano, Switzerland, 16-17 Oct. 2018, pp. 132141, https://doi.org/10.15221/18.132.

[4] W. Wright, "Man vs. Machine - Measuring People for the Apparel Industry", in Proc. of 3DBODY.TECH 2019 - 10th Int. Conf. and Exh. on 3D Body Scanning and Processing Technologies, Lugano, Switzerland, 22-23 Oct. 2019,doi:10.15221/19.033.

[5] IEEE Industry Connections 3D Body Processing (3DBP), https://standards.ieee.org/industryconnections/3d/bodyprocessing.html

[6] E. Scott, A.S.M. Sayem, "Landmarking and Measuring for Critical Body Shape Analysis Targeting Garment Fit", in Proc. of 3DBODY.TECH 2018 - 9th Int. Conf. and Exh. on 3D Body Scanning and Processing Technologies, Lugano, Switzerland, 16-17 Oct. 2018, pp. 222235,https://doi.org/10.15221/18.222.

[7] ISO 15535:2012 General requirements for establishing anthropometric databases.

[8] Eliasziw, M. et al.. "Statistical methodology for the concurrent assessment of interrater and intrarater reliability: using goniometric measurements as an example" in Physical therapy, 74(8), pp. 777-788, 1994.

[9] Gordon, C.C. et al., "2012 Anthropometric Survey of U.S. Army Personnel: Methods and Summary Statistics." Report No. NATICK/TR-15/007, 2015.

[10] ISO 20685:2010 3-D scanning methodologies for internationally compatible anthropometric databases. 